\title{
Enabling Practice Leaders to Reduce Patient Harm through "System-Base Practice and Practice-Based Learning and Improvement"
}

\author{
Ranjit Singh1,2 \\ ${ }^{1}$ Department of Family Medicine, Patient Safety Research Center, School of Medicine, State University of NY at \\ Buffalo, Buffalo, USA \\ ${ }^{2}$ School of Management, State University of NY at Buffalo, Buffalo, USA \\ Email: rs10@buffalo.edu, gsingh4@buffalo.edu
}

Received 1 September 2014; revised 24 October 2014; accepted 25 November 2014

Copyright $@ 2014$ by author and OALib.

This work is licensed under the Creative Commons Attribution International License (CC BY). http://creativecommons.org/licenses/by/4.0/

(c) (i) Open Access

\section{Abstract}

Background: Healthcare organizations regularly face new challenges. Their leaders must be adaptive through systematic approaches. These approaches must meet three basic needs: 1) facilitation of workflow/process/task assessment and improvement; 2) creation of high reliability organization; and 3) respect of each practice as a special part of the total healthcare system. The US Institute of Medicine has called for higher quality at lower cost through "leadership that fosters continuous learning". Retrospective methods are currently the most commonly used. These reveal only the tip of the iceberg of the total harm. The US Inspector General takes the view that the "current methods of detection of adverse events are inadequate". Recommended Approach: An innovative prospective process is put forward. It fosters empowerment and ownership, eventually leading to high reliability practices. Conclusion: This approach is shown to be effective in measuring safety state in a practice and reducing patient harm. Could this be the "better way" that the Inspector General is seeking? Our experience with this approach in the domains of medication safety, falls safety, postoperative pain, and assessment of effects of HIT introduction has demonstrated that it also promotes core competencies of "system-based practice and practice-based learning and improvement" in staff.

\section{Keywords}

Harm, Leadership, Practice, Reliability, Safety, System

Subject Areas: Health Policy, Internal Medicine 


\section{Introduction}

“First do no harm” call from Hippocrates (5th century BC), call for hygiene from Nightingale (1820-1910), precede the modern thrusts in this mission that include US Institute of Medicine (IOM) Report "to err is human" [1] and Donaldson's report “An Organization with a Memory” [2]. In 2004 WHO formed World Alliance for Patient Safety. The progress towards improvement has been very unsatisfactory. This calls for systems approach and practice-based management. Various accreditation authorities have started paying attention to this. For example, ACGME has recently called for implementation of "Next GME Accreditation System” [3], paying due attention to "System-Based Practice and Practice-Based Learning and Improvement".

In the outpatient settings alone, up to two hundred thousand preventable deaths occur per year [4] [5]. According to the Institute for Health Improvement (IHI), in the US healthcare eleven million patients are harmed every year. Primary care has a critical role in providing the health and well-being of communities across the nation. Reduction of harm is an ethical, societal and fiscal imperative. "Progress in 12 years after the Institute of Medicine's (IOM) calls for listening to patients and 50\% reduction of harm by 2005 has been disappointing” [6]. The US Inspector General of Health Human Services (HHS) has stated: "Current methods of detection of adverse events are inadequate and risk misdirection of present efforts to improve safety based quality”. Annual economic impact of harm to patients is estimated to be more than \$1 trillion. Literature on patient safety is relatively scarce [6]. Healthcare non-system needs major overhaul. A large proportion of US adults have had experience of harm caused by medical treatment. In the US consumer Union's view preventable adverse events are kept secret from them [7]. The WHO regards patient safety to be a Basic Human Right. It takes the view that healthcare should be a balm for human suffering and not a contributor to it [8]. Practice leaders must, therefore, play the appropriate role.

\section{Quality and Safety: Their Commonalities and Differences}

\subsection{Quality}

The quality of any health care practice/setting is its total system characteristic. It has seven dimensions and can be defined as the degree to which health services for individuals and populations deliver better health, better care, and better value, consistent with current professional knowledge. The seven dimensions are 1) Safety, 2) Timeliness, 3) Effectiveness, 4) Equity, 5) Efficiency, 6) Patient Centered, and 7) Continuity of care. The first six were adopted in the IOM 2001 report [9]. The needs expressed in the Patient Protection and Affordable Care Act must be addressed systematically so as to develop and assess innovative methods for inviting/engaging/ enabling all stakeholders, particularly patients.

\subsection{Safety}

It is one of the world's most pressing health care challenges. It can be defined as freedom from avoidable injuries. Its goal is to avoid, prevent and ameliorate adverse outcomes emanating from the care processes. It is important to state that " $a$ safe organization is a cost-effective quality organization" (Ex-Secretary of the Treasury, Ex-CEO of ALCOA and RAND, Paul O’Neill) [10]. "Partnership for Patients: Better Care Low Costs” and "Meaningful Use of Health Information Technology" are initiatives from the Department of Health and Human Services (HHS) designed to make care truly "patient-centered” [11].

Reason's trajectory of errors [12] aids understanding of the causes of failures in the form of situational (e.g. very unusual work load, power supply failure at critical juncture), latent (e.g. deficiencies in design, operation, maintenance, organisation and management) and active failures (e.g. human fallibility) that can result in adverse events, in the absence of appropriate technical (e.g. use of informatics, safe dosage packaging) and administrative (e.g. standard protocols, non-hierarchical team culture [13] [14]) barriers to this trajectory. The overall purpose is to create high reliability organization (HRO) [15].

\subsection{Commonalities}

Any quality health care setting has dimensions/components housed in a protective Donabedian "house of quality triad" of: "structure, process and outcome" [16] portrayed in Figure 1.

Structural quality expresses healthcare system capacities, how the system is configured, description of its 


\section{The House of Best Quality Practice}

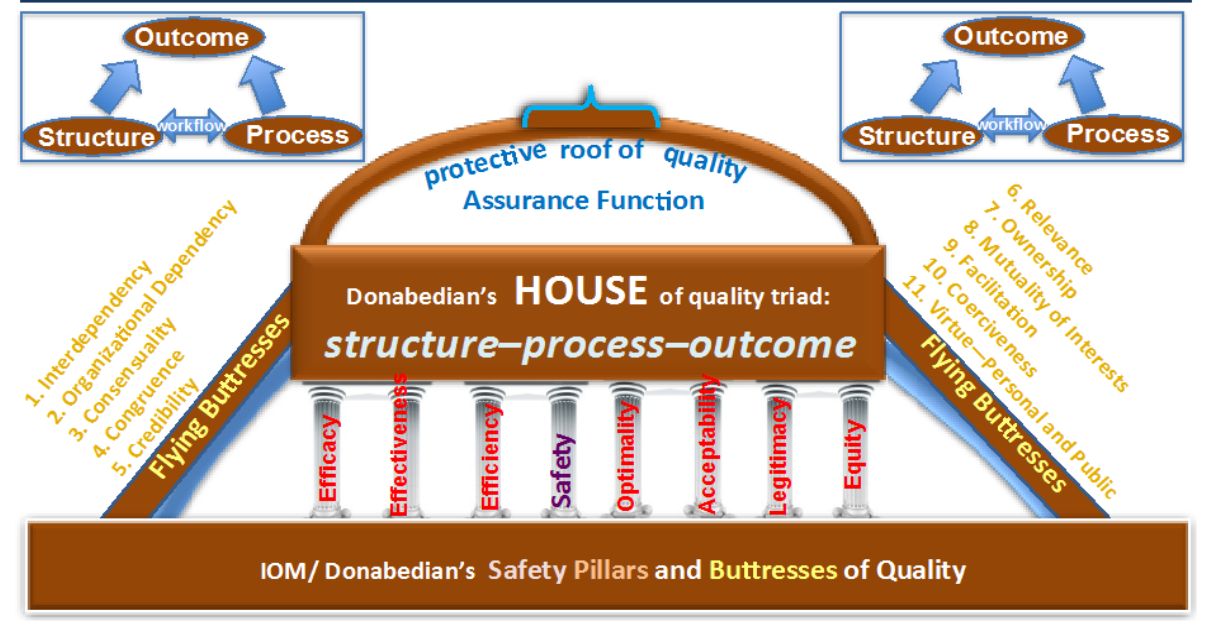

Figure 1. Donabedian's triad of structure-process-outcome [59].

components, and their inter-relationships are expressed. Organisational culture [13] [14] and stakeholder's satisfaction through their empowerment (with bottom-up approaches) are important elements.

Interactions between patients and clinicians as well as how care is delivered are through well designed work process [17] [18]. The best process measures should be based on evidence relating better process to better outcome (e.g. reliable testing processes reduce misdiagnosis-related harm).

Changes in health status of the patients and patient satisfaction are outcomes.

Any safe health care setting, whist being an indispensable and vital dimension of quality, has to be designed to face following systemic threats:

- Variability from patient to patient.

- Use of inappropriate time constraints.

- Lack of initiative to handle the unforeseen.

- Complexity of the process of care.

- Poor interfacing (e.g. transition between settings).

- Lack of error-preventing barriers.

- Inconsistency in the standards of care.

- Human fallibility-to err is human.

- Use of hierarchical in the system.

\section{Basic Approaches to Practice-Based Learning and Improvement for Harm Reduction}

Because healthcare organisations regularly face new challenges they must be adaptive to improve quality continually. Quality improvement in any setting has to be a systematic approach driven by experience and memory of all stakeholders and has to be data-informed. All improvement approaches must meet three basic needs: 1) treatment of each setting as a unique micro-system, 2) creation of high reliability organisation (HRO) [13]-[15], and 3) facilitation of workflow/process/task improvement [17] [18].

Apart from financial incentives (e.g. salary, capitation, fee for service, and pay for performance) that are fraught with shortcomings [19] [20] there are two basic approaches that have been used to reduce the burden of unsafe care. These are retrospective and prospective methods of safety assessment for management of improvement.

\subsection{Retrospective}

These include trigger tools, quality and safety indicators, internal and external audits, and error reports. Each 
one of these reveals only a tip of iceberg of quality gap and different perspectives of the same reality. These methods tend to be top-down and do not fully meet needs expressed earlier. Generalisations of the results from retrospective methods lead to dissatisfaction in stakeholders.

Even after the advent of PSOs, error reporting leads to both under-reporting (due to continued fear of repercussions) and abuse (examples of abuse include reports filed and counter-filed as a means of retaliation against colleagues). The culture of blame has been slow to change. As stated earlier, the Department of Health and Human Services' Inspector General (IG) has stated: "Current methods of detection of adverse events are inadequate and risk misdirection of present efforts to improve safety based quality" [21]. The IG has called for better ways of monitoring safety.

One of the main flaws in current methods is that they fail to incorporate the patient perspectives. There is worldwide awareness that patients are a primary source of information about these avoidable AEs [22]. We need to develop methods of inviting, engaging and enabling patients by creating a shared vision [23]-[25]. The Assistant Secretary for Health at Department of HHS (Koh et al. [26]) draws attention to the fact that patient engagement is the "blockbuster drug of the century" [24]. Reform has to happen at the point of delivery of healthcare [25]-[29]. Advantage should be taken of the AHRQ Guide to Patient and Family engagement and the Patient-reported outcome measures (PROMs) Report [30].

\subsection{Prospective}

This is designed to capture the memory [2] of all stakeholders so as to anticipate adverse events. Failure modes and effects analysis (FMEA) is the main method used in this category. It is recommended by IOM and the Joint Commission, recommending a series of steps based on FMEA [31] [32]. In ambulatory practices, however, this methodology is not feasible, particularly in underdeveloped countries [33].

\section{Proposed Model for Bottom-Up Humanistic Methodology}

\section{Introduction}

The reliability and sustainability of a FMEA lies in the fact that it is a bottom-up humanistic approach that reveals the rest of the iceberg [33]. As illustrated in Figure 2, it has to be built on five principles and three important considerations. This methodology contributes to building the structure-process-outcome triad (Figure 1).

This bottom-up systems approach [33]-[38], though based on FMEA, avoids the burden of resource requirements. It is designed to motivate and empower teams in any setting to assess quality baseline, identify most significant problems, devise and implement informed feasible solutions to prioritised issues, track changes and repeat the cycle continually. This 4-stage cyclic method is reproduced in Figure 3 from the author's earlier publication [13].

Stage (1) Measure current safety status with a confidential and anonymous on-line survey: An Instrument termed "Safety Enhancement and Monitoring Instrument-patient centered" (SEMI-P) is used for this. It is essential for all to understand the system of care in the setting. This is done by first identifying all the components and interlinks between them. This best done with a clear portrait of the practice [39] [40].

The survey includes a total of 140 different failure modes. These were listed after consultation with staff and patient advocates and review of literature [41]-[48]. This survey can be adapted to incorporate uniqueness of any practice.

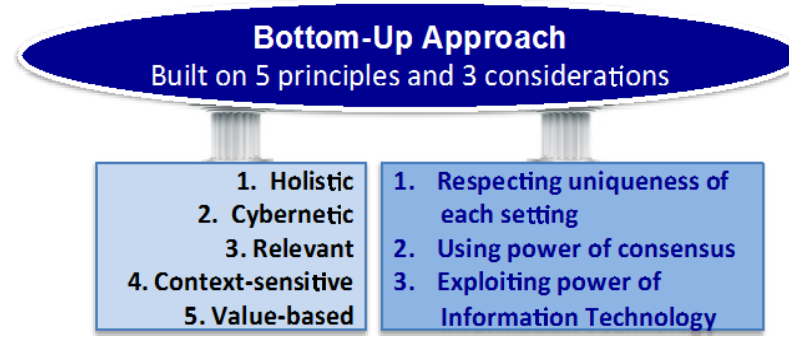

Figure 2. Structure of humanistic approach to change for harm reduction. 


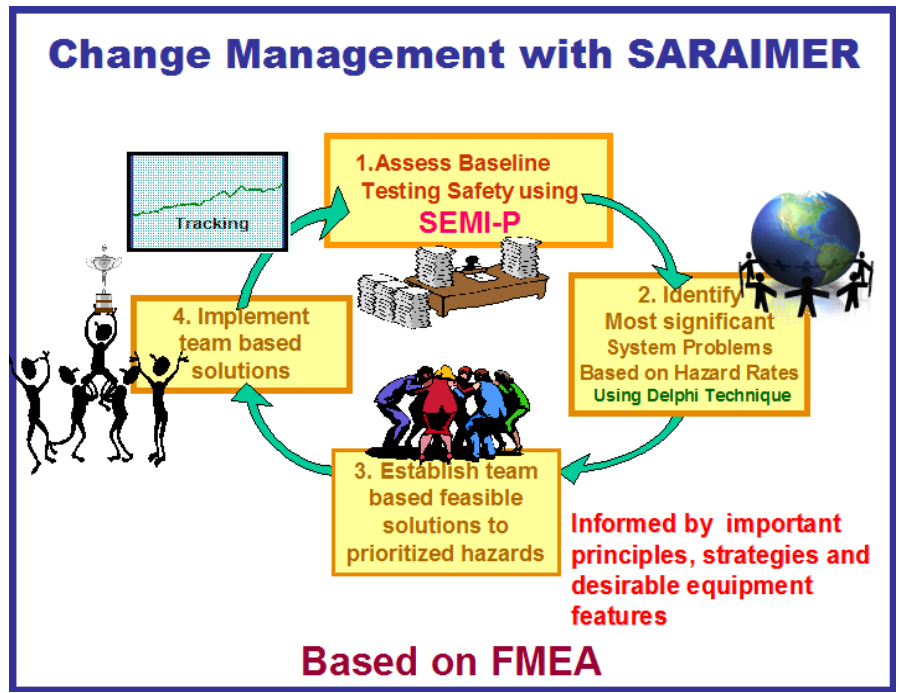

Figure 3. Bottom-up Error Reduction Intervention Cycle (ERIC) for system-based continuous learning [13].

Stage (2) Rank failure modes according to product of respective severity and frequency: In the survey, each respondent rates the frequency and severity of each error according to the categorical scales.

Hazard results are made available in visual format to help formation of consensus regarding prioritization.

Stage (3) Choose solutions to prioritized hazards: The solutions proposed are based on reliability and safety principles.

Stage (4) Implement interventions and monitor their effects: This is done with clear allocation of roles, responsibilities and time limits.

\section{Experience with Bottom-Up Methodology}

Supported by two AHRQ grants we have shown that all practice staff is able to collaborate fruitfully [33]-[38]. The methodology fosters culture of safety not only in the domains of primary care settings but also in hospital settings (post operative pain management [49] and falls reduction).

\section{Discussion}

The prevalent approaches are influenced by the 1911 book "The Principles of Scientific Management" [53] whereby only management is empowered to make decisions while workers are expected to follow unquestioningly.

The Error Reduction Intervention Cycle (ERIC) process illustrated in Figure 3 helps in forming shared goals [50]. Ownership and empowerment lead to worker and patient satisfaction and formation of reliable organizations [14] [15] [50]-[52].

Recent recommendations by international opinion leaders support and synergize with the author's approach as elaborated below.

In September 2012, IOM [54] called for higher quality at lower cost through "leadership that fosters continuous learning”. This report asserts that there can be no quality without safety. An August 2013 report [55] by Britain's National Advisory Group on the Safety of Patients in England to the British Prime Minister drew attention to the fact that the current state of patient safety is in "Crisis". It called for a "promise to learn and a commitment to act", reducing harm to patients by embracing wholeheartedly an ethic of learning. Both reports make the vital statement that the power of bottom-up commitment for continuous learning for quality improvement is, by far, much greater than that of the top-down efforts that are based on rules, standards and enforcement. Bottom-up initiatives and acts will help fulfill the mission of the US Patient Protection and Affordable Care Act (PPACA). We need to develop facilities that enable and help implementation of "A promise to learn-a commitment to act” [55]. Providers are generally frustrated with the top-down management methods that are by their 
very nature mechanistic (Taylorism) [53], as against humanistic (bottom-up) methods. Top-down methods include: practice profiles, quality and safety indicators, external audits, and trigger tools. These tools do not recognize the problems involved with defining and quantifying harm at the points of care.

\section{Conclusions}

Our very positive experience with the proposed approach in the domains of medication safety, falls safety, postoperative pain, and assessment of EMR introduction has demonstrated that it also promotes ACGME's core competencies [3] [57] [58] of "system-based practice and practice-based learning and improvement". Our objective is to prepare the minds [56] of teams and the individuals forming them, through our prospective methodology that invokes "Improvement Science" and refrains from reductionism.

The author's approach is supported by and synergizes with recent recommendations by international opinion leaders [54] [55].

\section{Acknowledgements}

The author is deeply indebted to AHRQ for funding our work. The support of his safety team is highly appreciated.

\section{References}

[1] Kohn, L.T., Corrigan, J. and Donaldson, M.S. (1999) To Err Is Human: Building a Safer Health System. National Academy Press, Washington DC.

[2] Donaldson, L. (2000) An Organisation with a Memory. Stationery Office, Great Britain.

[3] Nasca, T.J., Philibert, I., Brigham, T. and Flynn, T.C. (2012) The Next GME Accreditation System-Rationale and Benefits. Special Report. New England Journal of Medicine, 366, 1051-1056. http://dx.doi.org/10.1056/NEJMsr1200117

[4] Weingart, S.N., Wilson, R.M., Gibberd, R.W. and Harrison, B. (2000) Epidemiology of Medical Error. Western Journal of Medicine, 172, 390-393. http://dx.doi.org/10.1136/ewjm.172.6.390

[5] Starfield, B. (2000) Is US Health Really the Best in the World? Journal of the American Medical Association, 284, 483-485. http://dx.doi.org/10.1001/jama.284.4.483

[6] Balik, B. and Dobkiss, F. (2010) 10 Years after to Err Is Human: Are We Listening to Patients and Families Yet? Focus on Patient Safety, 13, 1-3.

[7] Denham, C.R. (2011) The Partnership with Patients: A Call to Action for Leaders. Journal of Patient Safety, 7, 113121. http://dx.doi.org/10.1097/PTS.0b013e31822d6f2a

[8] WHO (2008) Summary of Evidence on Patient Safety: Implications for Research. Working Group of World Alliance for Patient Safety.

[9] Institute of Medicine (2001) Crossing the Quality Chasm: A New Health System for the 21st Century. National Academy of Sciences, Washington DC.

[10] O’Neill, P. (2007) Op-Ed Contributor; a Health Care Bargain. New York Times, 16 October 2007.

[11] IOM (2012) Best Care at Lower Cost.

[12] Reason, J.T. (1990) Human Error. Cambridge University Press, Cambridge. http://dx.doi.org/10.1017/CBO9781139062367

[13] Singh, G., Singh, R., Thomas, E.J., et al. (2008) Measuring Safety Climate in Primary Care Offices. In: Henriksen, K., Battles, J.B., Keyes, M.A. and Grady, M.L., Eds., Advances in Patient Safety: New Directions and Alternative Approaches (Vol. 2: Culture and Redesign), Agency for Healthcare Research and Quality, Rockville, 59-72.

[14] Singh, R., Anderson, D., McLean, P.E., Rahega, D. and Singh, G. (2013) Interplay between Quantifiable and Unquantifiable Safety Climate as Affected by Successful Systems Approach to Medication Safety Improvement: Primary Care Settings. American Journal of Medicine and Medical Sciences, 3, 1-9.

[15] AHRQ (2008) Becoming a High Reliability Organization: Operational Advice for Hospital Leaders. Agency for Healthcare Research and Quality, Rockville.

[16] McDonald, K.M., Sundaram, V., Bravata, D.M., et al. (2007) Closing the Quality Gap: A Critical Analysis of Quality Improvement Strategies (Vol. 7: Care Coordination). Agency for Healthcare Research and Quality, Rockville. http://www.ncbi.nlm.nih.gov/books/NBK44015/ 
[17] Singh, R., et al. (2011) An Innovative Interactive Workflow Re-Design Tool Kit. Presented at NAPCRG 2011. http://www.fmdrl.org/index.cfm?event=c.beginBrowseD\&clearSelections=1\&criteria=Singh\#3580

[18] Singh, R., Singh, A., Raj, D. and Singh, G. (2013) Innovative Workflow and Process Improvement Toolkit to Ease and Enrich Meaningful Use of HIT: Example in Primary Care Office. Advances in Medical Education and Practice, 4, 231236.

[19] Roland, M. (2007) Does Family Medicine Have a Future? Plenary Section, NAPCRG. http://www.fmdrl.org/index.cfm?event=c.beginBrowseD\&clearSelections=1\&criteria=Martin\%20Niland\#1064

[20] Gillam, S.J. (2011) Pay for Performance in UK General Practice-The Ambiguous Impact of the Quality and Outcomes Framework. Expert Commentary, AHRQ, NQMC.

[21] Levinson, D.R. (2010) Adverse Events in Hospitals: National Incidence among Beneficiaries. Department of Health and Human Services, Office of Inspector General.

[22] Pronovost, P., Holzmueller, B.L.A., Ennen, C.S. and Fox, H.E. (2011) Overview of Progress in Patient Safety. American Journal of Obstetrics and Gynecology, 204, 5-10.

[23] Weingart, S.D. (2011) Patient-Reported Adverse Events: What Are We Waiting for? Joint Commission Journal on Quality and Patient Safety, 37, 494.

[24] Kish, L. (2013) The Blockbuster Drug of the Century: An Engaged Patient. HL7 Standards. http://www.hl7standards.com/blog/2012/08/28/drug-of-the-century/

[25] Simpson, P. (2013) Patient Voices: A Digital Library of Healthcare Stories. The Public Library of Science-Science Blog Network. PLOS Medicine Community Blog.

[26] Koh, K.K., Brach, C., Harris, M. and Parchman, M.L. (2013) A Proposed “Health Literate Care Model” Would Constitute a Systems Approach to Improving Patients’ Engagement in Care. Health Affairs, 32, 357-367. http://dx.doi.org/10.1377/hlthaff.2012.1205

[27] Conway, P.H. and Clancy, C. (2009) Transformation of the Health Care at the Front Line. Journal of the American Medical Association, 301, 763-765.

[28] Clancy, C.M. (2005) Evaluating the Potential of New Technology. Building a Better Delivery System: A New Engineering/Health Care Partnership. National Academies Press, Washington DC.

[29] Singh, R., Singh, A., Rowe, J., Singh, G. and Stott, E. (2012) Engaging Stakeholders in Reporting Adverse Drug Events with a Common Visual Language. Home Health Care Management Practice, 25, 54-61.

[30] Butt, Z. and Reeve, B. (2012) Enhancing the Patient's Voice: Standards in the Design and Selection of Patient-Reported Outcomes Measures (PROMs) for Use in Patient-Centered Outcomes Research. Submitted to Patient Centeredness Workshop, PCORI Methodology Committee.

[31] JCAHO (2001) Comprehensive Accreditation Manual for Hospitals: The Official Handbook, Update 1. Joint Commission Resources, Oak Brook,

[32] Marder, R. and Sheff, R.A. (2002) The Step-by-Step Guide to Failure Modes and Effects Analysis. Opus Communications, Inc., Marblehead.

[33] Singh, R., Singh, A., Taylor, J.S., Rosenthal, T.C., Singh, S. and Singh, G. (2006) Building Learning Practices with Self-Empowered Teams for Improving Patient Safety. Journal of Health Management, 8, 91-118. http://dx.doi.org/10.1177/097206340500800107

[34] Singh, R., Anderson, D.R., McLean-Plunkett, E.A., Brooks, R., Wisniewski, A. and Singh, G. (2012) IT-Enabled Systems Engineering Approach to Monitoring and Reducing ADEs. The American Journal of Managed Care, 18, 169175.

[35] Singh, R., Anderson, D.R., McLean-Plunkett, E.A., Wisniewski, A., Kee, R., Fox, C., Gold, K. and Singh, G. (2012) Effects of Self-Empowered Teams on Rates of Adverse Drug Events in Primary Care. International Journal of Family Medicine, 2012, Article ID: 374639.

[36] Singh, R., Singh, A., et al. (2011) IT-Based Systems Approach to Engaging Patients in Medical Error Reporting: In Concurrence with Staff. Proceedings of the 17th European Concurrent Engineering Conference, London, 18-20 April 2011.

[37] Singh, R., Singh, A., Anumba, C., et al. (2011) Enhancing and Measuring Patient Safety with an Innovative WebBased Systems Approach. International Journal of Advanced Intelligence Paradigms, 3, 164-177. http://dx.doi.org/10.1504/IJAIP.2011.039747

[38] Economist (2013) Making Pay Work: Why Bosses Should Be Careful When Using Performance-Related Pay. The Economist, 25 May 2013.

[39] Singh, R., Singh, A., et al. (2011) Creating a Common Vision for All Stakeholders to Make Healthcare Safer with Interactive Visual Monitoring. International Journal of Advanced Intelligence Paradigms, 3. 
[40] Singh, R., Pace, W., Singh, A., Fox, C. and Singh, G. (2008) A Visual Computer Interface Concept for Making Error Reporting Useful at the Point of Care. In: Henriksen, K., Battles, J.B., Keyes, M.A. and Grady, M.L., Eds., Advances in Patient Safety: New Directions and Alternative Approaches (Vol. 1), Agency for Healthcare Research and Quality, Rockville, 307-320.

[41] Kohn, L.T., Corrigan, J. and Donaldson, M.S. (2000) To Err Is Human: Building a Safer Health System. National Academy Press, Washington DC.

[42] Dovey, S.M., Meyers, D.S., Phillips Jr., R.L., et al. (2002) A Preliminary Taxonomy of Medical Errors in Family Practice. Quality \& Safety in Health Care, 11, 233-238. http://dx.doi.org/10.1136/qhc.11.3.233

[43] Makeham, M.A.B., Dovey, S.M., County, M. and Kidd, M.R. (2002) International Taxonomy of Errors in General Practice: A Pilot Study. The Medical Journal of Australia, 177, 68-72.

[44] Sheikh, A. and Hurwitz, B. (2001) Setting up a Database of Medical Error in General Practice: Conceptual and Methodological Considerations. British Journal of General Practice, 51, 57-60.

[45] Bhasale, A. (1998) The Wrong Diagnosis: Identifying Causes of Potentially Adverse Events in General Practice Using Incident Monitoring. Family Practice, 15, 308-318. http://dx.doi.org/10.1093/fampra/15.4.308

[46] Britt, H., Miller, G.C. and Steven, I.D. (1997) Collecting Data on Potentially Harmful Events: A Method of Monitoring Incidents in General Practice. Family Practice, 14, 101-106. http://dx.doi.org/10.1093/fampra/14.2.101

[47] Ely, J., Levinson, W., Elder, N.C., Mainous, A. and Vinson, D. (1995) Perceived Causes of Family Physicians Errors. Journal of Family Practice, 40, 337-344.

[48] Fischer, G., Fetters, M.D., Munro, A.P. and Goldman, E.D. (1997) Adverse Events in Primary Care Identified from a Risk-Management Database. Journal of Family Practice, 45, 40-46.

[49] Singh, R., Naughton, B., Anderson, D.R. and Singh, G. (2008) Building Self-Empowered Teams for Improving Safety in Post-Operative Pain Management. In: Henriksen, K., Battles, J.B., Keyes, M.A. and Grady, M.L., Eds., Advances in Patient Safety: New Directions and Alternative Approaches (Vol. 3: Performance and Tools), Agency for Healthcare Research and Quality, Rockville, 37-50.

[50] Kotter, J. (1996) Leading Change. Harvard Business School Press, Cambridge.

[51] Senge, P. (1990) The Fifth Discipline: The Art and Practice of Learning Organization. Doubleday, New York.

[52] Wheatley, M. (1992) Leadership and the New Science: Learning about Organizations from an Orderly Universe. Berret-Koehler Publishers, San Francisco.

[53] Taylor, F.W. (1911) The Principles of Scientific Management. Harper and Brothers Publishers, New York.

[54] IOM (2012) Best Care at Lower Cost: The Path to Continuously Learning Health Care in America.

[55] National Advisory Group on the Safety of Patients in England (2013) A Promise to Learn—A Commitment to Act. National Health Service, UK.

[56] Singh, R., Hickner, J., Mold, J. and Singh, G. (2014) Chance Favors Only the Prepared Mind: Preparing Minds to Systematically Reduce Hazards in the Testing Process in Primary Care. Journal of Patient Safety, 10, 20-28.

[57] Singh, R., Naughton, B., Taylor, J.S., et al. (2005) A Comprehensive Collaborative Patient Safety Residency Curriculum to Address the Core Competencies. Medical Education, 39, 1195-1204. http://dx.doi.org/10.1111/j.1365-2929.2005.02333.x

[58] Singh, R., Singh, A., Fish, R., McLean, D., Anderson, D.R. and Singh, G. (2009) A Patient Safety Objective Structured Clinical Exam (OSCE). Journal of Patient Safety, 5, 55-60. http://dx.doi.org/10.1097/PTS.0b013e31819d65c2

[59] Schiff, G.D. and Rucker, T.D. (2001) Beyond Structure-Process-Outcome: Donabedian’s Seven Pillars and Eleven Buttresses of Quality. The Joint Commission Journal on Quality Improvement, 27, 169-174. 